\title{
Nanoscale Chemical Imaging of Coadsorbed Thiolate Self-assembled Monolayers on Au(111) by Tip-Enhanced Raman Spectroscopy
}

\author{
Feng Shao, ${ }^{1,2 *}$ Liqing Zheng, ${ }^{2}$ Jinggang Lan, ${ }^{3}$ Renato Zenobi ${ }^{2 *}$ \\ ${ }^{1}$ Department of Physics and Astronomy, National Graphene Institute, University of Manchester, \\ Manchester, M13 9PL, UK \\ ${ }^{2}$ Department of Chemistry and Applied Biosciences, ETH Zurich, 8093 Zurich, Switzerland \\ ${ }^{3}$ Department of Chemistry, University of Zurich, 8057 Zurich, Switzerland \\ *Corresponding Authors: feng.shao@manchester.ac.uk; zenobi@org.chem.ethz.ch
}

\begin{abstract}
Self-assembled monolayers (SAMs) of thiolates on metal surfaces are of key importance for engineering surfaces with tunable properties. However, it remains challenging to understand binary thiolate SAMs on metals at the nanoscale under ambient conditions. Here we employ tip-enhanced Raman spectroscopy (TERS) and density functional theory (DFT) calculations to investigate local information of binary SAMs on $\mathrm{Au}(111)$ coadsorbed from an equimolar mixture of $p$-cyanobenzenethiol (pCTP) and $p$-aminothiophenol (pATP), including chemical composition, coadsorption behavior, phase segregation, plasmon-induced photocatalysis, and solvation effects. We found that upon competitive adsorption of pCTP and pATP on Au(111) from a methanolic solution, the coadsorption initially occurs randomly and homogeneously; eventually, pATP is replaced by pCTP through gradual growth of pCTP nanodomains. TERS imaging also allows for visualization of the plasmon-induced coupling of pATP to $p, p$ 'dimercaptoazobenzene (DMAB) and the solvation-induced phase segregation of the binary SAMs into nanodomains, with a spatial resolution of $\sim 9 \mathrm{~nm}$ under ambient conditions. According to DFT calculations, these aromatic thiolates differing only in their functional groups, $-\mathrm{CN}$ versus $-\mathrm{NH}_{2}$, show different adsorption energy on $\mathrm{Au}(111)$ in vacuum and
\end{abstract}


methanol, and thus the solvation effect on adsorption energy of these thiolates in methanol can determine the dispersion state and replacement order of the binary thiolates on $\mathrm{Au}(111)$.

\section{INTRODUCTION}

Formation of self-assembled monolayers (SAMs) provide a flexible and convenient strategy to tailor interfacial properties of metals or metal oxides, ${ }^{1}$ which is crucial in various research fields, e.g., molecular biology, interface chemistry, organic electronics, and materials science. ${ }^{2,3}$ In particular, the S-Au covalent bond between gold and sulfur makes thiolate SAMs inherently robust but modifiable on $\mathrm{Au}$ surfaces, and thus thiolate SAMs technologically become an attractive method for tunable surface engineering. ${ }^{2,3}$ The coadsorption of binary thiolate SAMs on $\mathrm{Au}$ surfaces, either with homogeneously mixed dispersions or with locally separated domains, is able to facilitate the further tuning of surface work function, resonance features, electrical transport, thermal conductance, and catalytic activities. ${ }^{4-8}$ For example, by changing their mixture ratios, the homogeneously mixed binary SAMs of non-substituted (H-BPT) and fluorine-substituted (F-BPT) mercaptobiphenyls on $\mathrm{Au}(111)$ enable alterable-charge tunneling

rates in molecular junctions. ${ }^{5}$ If 1 -octanethiol and 6-mercaptohexanol binary SAMs form nanodomains on flat Au surfaces, their interfacial energies show a significant dependence on average composition. ${ }^{9}$ Moreover, for molecular electronics, the mixing of components in SAMs will result in more diversity and complexity of molecular-scale electronic devices, and thus their structures and functions may be extended in a nearly unlimited fashion. ${ }^{10}$ An in-depth understanding of binary thiolate SAMs on metals will help us design thiolates with desired functional groups, mixture ratios, assembly behavior, and displacement order, which in turn extends these SAMs to more customized applications. 
Conventional spectroscopic methods, such as X-ray photoelectron spectroscopy (XPS) and near-edge X-ray absorption fine structure (NEXAFS) spectroscopy, ${ }^{11-13}$ have been used to deduce surface composition and charge states of binary SAMs, and to assess orientation differences between pure and mixed thiolate SAMs. Although rich chemical information is available, these spectroscopic approaches fail to provide sufficient lateral resolution to distinguish separation phases within binary SAMs at the nanoscale. Alternatively, scanning probe microscopy (SPM), such as scanning tunnelling microscopy (STM) and atomic force microscopy (AFM), ${ }^{14-19}$ are able to clearly reveal assembly patterns, domain boundaries, striped phases, and steric interactions of SAMs with (near-)atomic resolution. ${ }^{17-19}$ However, these methods fail to discern different SAMs based on their chemical composition; and even monothiolate SAMs can form various patterns on $\mathrm{Au}(111) .^{20}$

Tip-enhanced Raman spectroscopy (TERS) has emerged as a promising tool for the investigation of thiolate SAMs, by integrating Raman spectroscopy with SPM to allow for acquiring Raman fingerprint information and nanometer spatial resolution simultaneously. ${ }^{21-24}$ Early studies on SAMs were reported by Picardi et al., ${ }^{25}$ where TERS signals of an azobenzene thiol monolayer on $\mathrm{Au}(111)$ were found to be strongly dependent on the tunneling parameters and incident light polarization. Further investigations of binary thiolate SAMs by TERS imaging were carried out on isomeric 2-mercaptopyridine/4-mercaptopyridine, ${ }^{26} 1$ decanethiol/[4-(phenylazo)phenoxy]hexane-1-thiol, ${ }^{27}$ benzenethiol/oligomeric phenyleneethynylene thiol, ${ }^{28}$ and benzenethiol/benzenemethanethiol mixed systems. ${ }^{29,} 30$ Localized information of these mixed thiolate SAMs, e.g., specific relative composition, molecular replacement behavior, surface morphological changes, and phase segregation, could generally be observed. However, the best spatial resolution of TERS imaging for binary SAMs is still limited to larger than 15 nanometres under ambient conditions, ${ }^{28,29}$ which is not adequate for 
chemically resolving smaller nanodomains and thus providing more insights into the behavior of coadsorption on $\mathrm{Au}(111) .{ }^{25-30}$ Besides, previous TERS studies did not provide suggestion on how solvents affect adsorption energy of thiolates and induce phase segregation (i.e., solvation effects) during binary SAMs formation on $\mathrm{Au}(111){ }^{25-30}$

In this study, we present full spectroscopic TERS imaging of coadsorbed binary thiolate SAMs on $\mathrm{Au}(111)$ with a spatial resolution $\sim 9 \mathrm{~nm}$ under ambient conditions. Two structurally similar but spectrally identifiable aromatic thiolates, $p$-cyanobenzenethiol (pCTP) and $p$ aminothiophenol (pATP), are chosen as coadsorption competitors on $\mathrm{Au}(111)$ from an equimolar solution. Although their close molecular lengths hinder them from being distinguished by STM imaging, their different tail groups $\left(-\mathrm{CN}\right.$ versus $\left.-\mathrm{NH}_{2}\right)$ facilitate TERS analysis of the chemical composition, such that information about coadsorption behavior, phase segregation, solvation effect, and plasmon-induced photocatalysis of the mixed SAMs on $\mathrm{Au}(111)$ can be obtained. Additionally, TERS imaging visualizes the coadsorption competition over time and discerns pure pCTP nanodomains and binary nanodomains formed in the mixed SAMs, with a domain size down to $<10 \mathrm{~nm}$. Combined with density functional theory (DFT) calculations, we found that solvation effects can alter the thiolate adsorption energies on $\mathrm{Au}(111),{ }^{31-33}$ and thus pCTP would gradually replace pATP on the surface during the coadsorption time. Our findings indicate that TERS appears to be an attractive method for studying binary SAMs on atomically flat surfaces at the nanometer scale and molecular level under ambient conditions.

\section{EXPERIMENTAL SECTION}

\section{Chemicals and Materials}


Clean $\mathrm{Au}(111)$ on mica $(4 \times 4 \mathrm{~mm}$, Phasis, Switzerland) was used as received. $p$ cyanobenzenethiol (pCTP, $\geqslant 95.0 \%$, Apollo Scientific Ltd, UK) and $p$-aminothiophenol (pATP, $\geqslant 97.0 \%$, Sigma-Aldrich, Switzerland) were used as received without any further purification. The single-component solutions $(1.0 \mathrm{mM})$ of pCTP and pATP were prepared in methanol (99.9\%, Sigma-Aldrich, Switzerland). The mixed solution was prepared with an equimolar mixture (1:1) of the above pure pCTP and pATP solutions. Silver wires (diameter $0.25 \mathrm{~mm}, 99.9985 \%$, Alfa Aesar, Germany) were electrochemically etched as STM/TERS probes. A 3:1 (v/v) mixture of ethanol (absolute, Sigma-Aldrich, Switzerland) and perchloric acid (70\%, Sigma-Aldrich, Switzerland) was used as the etchant. A potential of $8 \mathrm{~V}$ was applied, and a current of $\approx 10 \mathrm{~mA}$ was measured during the etching process, as described in our previous work. ${ }^{34-36}$ Note that the etchant is potentially a highly explosive mixture under heat if metal traces are present. A laboratory fume hood and personal protective equipment (PPE) are required at work.

\section{Preparation of SAMs}

Pure SAMs of either pCTP or pATP were prepared by immersing the clean Au(111) into corresponding methanol solutions $(1.0 \mathrm{mM})$ for $10 \mathrm{~min}$. Binary SAMs of pCTP/pATP were prepared by a coadsorption method. The individual $\mathrm{Au}(111)$ substrates were immersed into the mixed solution with an equimolar mixture (1:1) for different times: $10 \mathrm{~min}, 1 \mathrm{~h}, 2 \mathrm{~h}$, and $6 \mathrm{~h}$. Before ex-situ SEM and TERS imaging, all the corresponding SAMs/Au(111) surfaces were thoroughly washed by pure methanol to remove the physically adsorbed molecules, and then were dried under nitrogen blow. 


\section{STM and TERS Measurements}

All ex-situ STM and TERS images were acquired on a combined STM/Raman microscope (Ntegra Spectra, NT-MDT, Zelenograd, Russia) which was enclosed by a homemade acoustic isolation box and operated under ambient conditions. ${ }^{34-36}$ The instrument is equipped with an air objective $(100 \times, \mathrm{NA}=0.7$, Mitutoyo, Japan $)$ and an electron-multiplying charge-coupled device (EMCCD, Newton 971 UVB, Andor, Belfast, UK). The spectrometer was calibrated by a standard neon lamp (Renishaw, UK). TERS maps were collected in the STM feedback (constant current mode), with the sample surface moved by the piezo stage in the $x, y$, and $z$ directions while keeping the relative laser-to-tip position fixed. The dynamic range of the piezo scanner $(6.0 \mu \mathrm{m} \times 6.0 \mu \mathrm{m}$ in $x y$ and $2.0 \mu \mathrm{m}$ in $z$ with $220 \mathrm{~V})$ is small, which ensures highly precise control of the tip-substrate gap and $x y$ coordinate with a $<0.1 \mathrm{~nm}$ step size. An exposure time of $1 \mathrm{~s}$ and 1 accumulation were used for all spectra and images collection. A $632.8 \mathrm{~nm}$ HeNe laser at an incident power of $0.5 \mathrm{~mW}$ was used to illuminate the $\mathrm{Ag}$ tip/Au(111) substrate configuration. This gap-mode TERS geometry enables higher field enhancement due to electromagnetic coupling between the Ag tip and the Au substrate under $632.8 \mathrm{~nm}$ laser excitation. ${ }^{36,37}$ During TERS measurements, the bias voltage was set to $0.1 \mathrm{~V}$, and the tunneling current was kept at $1.0 \mathrm{nA}$. In order to obtain STM images with higher resolution, $256 \times 256$ line scans were applied before TERS mapping. All TERS images (over a $100 \times 100 \mathrm{~nm}^{2}$ area with $32 \times 32$ pixels) were obtained after overnight measurements (continuous laser illumination and data recording) to minimize the thermal drift of the system. The cleanliness of the Ag tip was examined with a fresh $\mathrm{Au}(111)$ surface after imaging to ensure that the obtained TERS signals come from the SAMs instead of coming from molecules adsorbed on the tip. Spectral processing followed previously described procedures. ${ }^{34-36}$ 


\section{DFT calculations}

Theoretical Raman spectra were calculated using the Gaussian 09 software (Gaussian, Wallingford, USA) based on the DFT method. ${ }^{38,39}$ All calculations, including full geometry optimizations and frequency predictions, were performed using the B3LYP/6-311+G(d) basis set. While for Au metal atoms, the valence electrons and the inner shells were described by the LANL2DZ basis set. ${ }^{38}$ All calculated frequencies were scaled with proper factors (ca. 0.98) compared to confocal Raman spectra. The optimized geometries and the calculated vibrational modes were visualized using the Gaussview 6 package. ${ }^{35}$

Adsorption energy $\left(\Delta \boldsymbol{E}_{\text {ads }}\right)$ of the thiolates on $\mathrm{Au}(111)$ were calculated by using the $\mathrm{CP} 2 \mathrm{~K}$ package. $^{40,41}$ The PBE exchange functional ${ }^{42}$ with Grimme D3 correction $^{43}$ was used to describe the interfacial system. Kohn-Sham DFT was used as the electronic structure method in the framework of the Gaussian and plane-wave (GPW) method. ${ }^{44}$ The Goedecker-TeterHutter (GTH) pseudopotentials and DZVP-MOLOPT-SR-GTH basis sets were utilized to describe the surfaces and molecules, as well as to treat valence-core interactions with $11,6,5$, 4, and 1 valence electrons for $\mathrm{Au}, \mathrm{S}, \mathrm{N}, \mathrm{C}$, and $\mathrm{H}$, respectively. ${ }^{45,}{ }^{46}$ A plane-wave energy CUTOFF with 400 Rydberg have been employed. Fermi-Dirac smearing was applied to study the metallic system, the electron temperature was set to $300 \mathrm{~K}$. Au metal slab containing 144 metal atoms was shaped to obtain $(111)$ surfaces with four $(6 \times 6)$ atomic layers, and the $1 / 4$ monolayer coverage $(3 \times 3)$ of thiolates was used for the calculations. To decouple the interaction between two neighboring images along the Z-direction, ${ }^{47}$ a $15 \AA$ of vacuum layer was placed on top of the studied surface slabs (Figure S1). In order to take solvation effects into account, the self-consistent continuum solvation (SCCS) model was applied for comparison. ${ }^{48-50}$ As an implicit solvation method that treats solvent as a structureless 
continuous medium, the SCCS model can provide a statistical and average effect of the bulk solvent and improve piecewise defined dielectric model function. ${ }^{41,48}$ A CD5 method was used to calculate the numerical derivatives on the real-space grids. An ANDREUSSI method was used to define the maximum and minimum density values of the dielectric smoothing function, representing the dielectric constant. ${ }^{48}$ A dielectric constant $(\varepsilon)$ of 32.7 was used in the bulk solvent. ${ }^{51}$ All geometries and configurations were fully relaxed and optimized until the maximum force on each atom was less than $0.05 \mathrm{eV} / \AA$. All of the simulations were carried out by keeping the two bottom metal layers fixed at the initial coordinates to maintain the bulk behavior of the inner part of the slab. The adsorption energy ( $\left.\Delta \boldsymbol{E}_{\mathrm{ads}}\right)$ of individual pCTP and pATP molecules on $\mathrm{Au}(111)$, both in vacuum and methanol at various coverages, was calculated using Equation (1): ${ }^{52}$

$$
\Delta E_{\text {ads }}=\frac{1}{n}\left(E_{\text {complex }}-E_{\text {slab }}-n \times E_{\text {thiol }}-\frac{n}{2} E_{\mathrm{H}_{2}}\right)
$$

where $\boldsymbol{E}_{\text {complex }}$ is the total energy of the thiol-Au(111) complex models, $\boldsymbol{E}_{\text {slab }}$ is the total energy of the optimized $\mathrm{Au}(111)$ slab, $\boldsymbol{E}_{\text {thiol }}$ is the energy of the optimized thiol molecules, $\boldsymbol{E}_{\mathbf{H}_{2}}$ is the $\mathrm{H}_{2}$ formation energy, and $\boldsymbol{n}$ is the number of the thiol molecules in the system.

\section{RESULTS AND DISCUSSION}

\section{The choice of pATP and pCTP}

Two structurally similar aromatic thiolates, pCTP and pATP, were chosen to form binary SAMs on Au(111) (Figure S2, see Supplementary Information) based on the coadsorption method, as shown in Figure 1a. They both have a benzenethiol skeleton functionalized with slightly different tail groups, $-\mathrm{CN}$ versus $-\mathrm{NH}_{2}$. This results in a very close molecular length for pCTP $(7.178 \AA)$ and pATP $(6.538 \AA$, Figure S3), respectively. In contrast to previous 
alkanethiol binary SAMs with distinctly different lengths, ${ }^{26-28}$ topographic imaging by AFM or STM can hardly identify the pCTP/pATP components in a mixed SAMs under ambient conditions. However, their $-\mathrm{CN}$ and $-\mathrm{NH}_{2}$ tail groups are chemically distinct, such that differences in the vibrational spectroscopy, photocatalytic activity, and solvation effect are expected. Accordingly, TERS imaging is capable of overcoming the aforementioned technical hurdle since it simultaneously provides Raman fingerprint information and nanometer spatial resolution (Figure 1b).
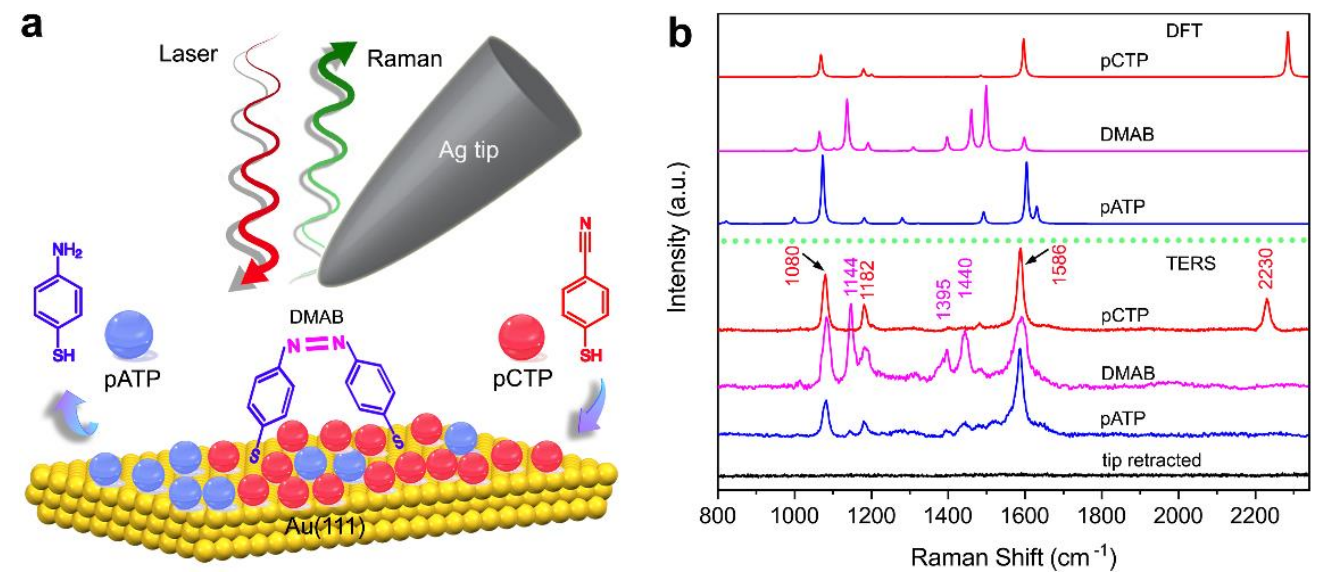

Figure 1. (a) Schematic illustration of gap-mode TERS study of a pATP/pCTP binary SAM on $\mathrm{Au}(111)$. (b) Representative experimental (lower half) and calculated (upper half) spectra of pCTP (red curves), pATP (blue curves), and DMAB (purple curves).

In the gap-mode TERS configuration (Figure S4), the Ag tip plays a triple role: (i) as Raman signal booster enabling highly sensitive detection of the different chemical entities in a binary SAMs; (ii) as tunneling probe enabling high spatial resolution imaging of the surface topography; and (iii) as single-particle photocatalyst enabling plasmon-mediated coupling reaction of pATP. ${ }^{53}$ The plasmon-driven conversion of pATP to $p, p^{\prime}$-dimercaptoazobenzene (DMAB) is one of the most well studied model reactions in thiolate SAMs.${ }^{54}$ Compared to the initial pATP peaks at 1080, 1182, and $1586 \mathrm{~cm}^{-1}$ (Figure 1b), three characteristic Raman peaks at 1144,1395 , and $1440 \mathrm{~cm}^{-1}$ present in the new DMAB spectrum after extending the laser 
irradiation time to ca. $5 \mathrm{~s}$ (for more details, see Figure S5). Notably, the appearance of DMAB signals implies the surrounding condition of pATP within the binary SAMs on Au(111). This is because the formation of DMAB needs the plasmon-induced coupling between two atomically adjacent pATP molecules, while two neighbouring pATP and pCTP molecules cannot react with each other. Moreover, due to the stability of the $-\mathrm{CN}$ group in plasmon hotspots and the explicit $\mathrm{C} \equiv \mathrm{N}$ stretching mode at $2230 \mathrm{~cm}^{-1}$, pCTP has been selected as another participant in our binary SAMs. All of the Raman peak assignments were supported by DFT calculations (Figure S6 and Table S1), which are in good agreement with the experimental spectra (Figure 1b). Note that the relative intensities of simulated spectra are different from the corresponding TERS results, which is probably due to a lack of consideration of charge transfer effects and surface selection rules in the simplified DFT calculations (Figure S6 and Table S1). ${ }^{37,55-57}$

\section{STM and TERS imaging of single-component SAMs}

Pure SAMs on $\mathrm{Au}(111)$ were prepared by chemisorption of either pCPT or pATP thiolates (Figures 2a, d). Individual, fresh $\mathrm{Au}(111)$ substrates were immersed into corresponding methanol solutions $(1.0 \mathrm{mM})$ for $10 \mathrm{~min}$ at room temperature. Before TERS imaging, the morphologies of these pure SAMs on Au(111) were determined by STM at ambient conditions. By selecting Ag tips and controlling the STM parameters (Figure S7), Au(111) atomic terraces can be observed from both substrates with pCTP or pATP pure SAMs under ambient conditions (Figures 2a, d). However, compared to high-resolution STM imaging of a bare Au(111) using a Pt-Ir tip (Figure S2), these substrates become rougher even only after 10 mins immersion in the thiolate solutions. This stems from the fact that thiolates are able to strongly modify the initial surface and pull out $\mathrm{Au}$ atoms during their self-assembling on $\mathrm{Au}(111) .{ }^{58}$ 
To offset fluctuations (e.g., due to variations in laser power or tip-sample distance) in the TERS enhancement during the imaging, TERS intensity ratio maps were evaluated to assess the molecular component and orientation distribution of the pure SAMs, ${ }^{34}$ as reported in Figures $2 b$, e. The intensities of the representative $\mathrm{C} \equiv \mathrm{N}$ stretching mode at $2230 \mathrm{~cm}^{-1}$ for pCTP and the $\mathrm{N}=\mathrm{N}$ stretching mode at $1440 \mathrm{~cm}^{-1}$ for DMAB, respectively, were divided by the intensity of the $1586 \mathrm{~cm}^{-1}$ band, which is the shared benzene ring deformation mode (Table $\mathrm{S} 1$ ). These maps were obtained from a $100 \times 100 \mathrm{~nm}^{2}$ area with $3.1 \times 3.1 \mathrm{~nm}^{2}$ pixels resolution (Figures 2b, e). Except for a few unoccupied or unreacted sites (dark blue pixels in Figures 2b, e), the $\mathrm{Au}(111)$ surface was completely covered by pCTP or pATP SAMs after a 10-min immersion time. Furthermore, changes in molecular orientation are revealed by the intensity ratio maps (Figures $2 \mathrm{~b}$, e), since the TERS intensity ratio is sensitive to the molecular adsorption configuration and orientation on a flat metal substrate. ${ }^{34}$ In particular, pATP will quickly convert into DMAB (ca. 5 s, Figure S5) during TERS mapping through plasmoninduced photocatalysis, and the pATP signal is only visible at the beginning of the mapping, as indicated by the dark blue pixels in Figure 2e. However, the "hot" pixels with a peak ratio ( $I_{1440}$ to $\left.I_{1586}\right)$ greater than 0.3 suggest the generation of DMAB on $\mathrm{Au}(111)$ (for more details, see Figure S5). Note that since the TERS maps are recorded from bottom to top (Figure 2e), the upper half part of the map allows for the photocatalytic conversion even longer, and thus stronger DMAB peak ratios $\left(I_{1440}\right.$ to $\left.I_{1586}\right)$ at the latter half of the map can be observed (Figure S4).

Typical TERS spectra of pCTP and pATP/DMAB were recorded at the locations in the maps marked with numbers (Figures 2c, f). The color coding in Figure 2 represents how frequently 
each type of spectrum appears in the maps. The vibrational modes of these thiolates on $\mathrm{Au}(111)$ were simulated and assigned via DFT calculations (Table S1). Besides the benzene ring inplane stretching mode at $1586 \mathrm{~cm}^{-1}$, the two thiolates have two more common vibrational modes: the $\mathrm{C}-\mathrm{S}$ stretching mode $(v)$ at $1080 \mathrm{~cm}^{-1}$ and the in-plane $\mathrm{C}-\mathrm{H}$ bending mode $(\delta)$ at $1182 \mathrm{~cm}^{-1}$ (Figure 2c). ${ }^{53}$ Three feature peaks at 1144, 1395, and $1440 \mathrm{~cm}^{-1}$ appear, which can be ascribed to the $\mathrm{C}-\mathrm{N}$ stretching mode $\left(1144 \mathrm{~cm}^{-1}\right)$ and the $\mathrm{N}=\mathrm{N}$ stretching mode (1395 and $1440 \mathrm{~cm}^{-1}$ ) of DMAB, respectively (Table S1). In contrast, the $\mathrm{C} \equiv \mathrm{N}$ stretching mode at 2230 $\mathrm{cm}^{-1}$ provides an unequivocal marker band for pCTP to chemically distinguish it from pATP or DMAB in the mixed thiolate adlayers by TERS.

a
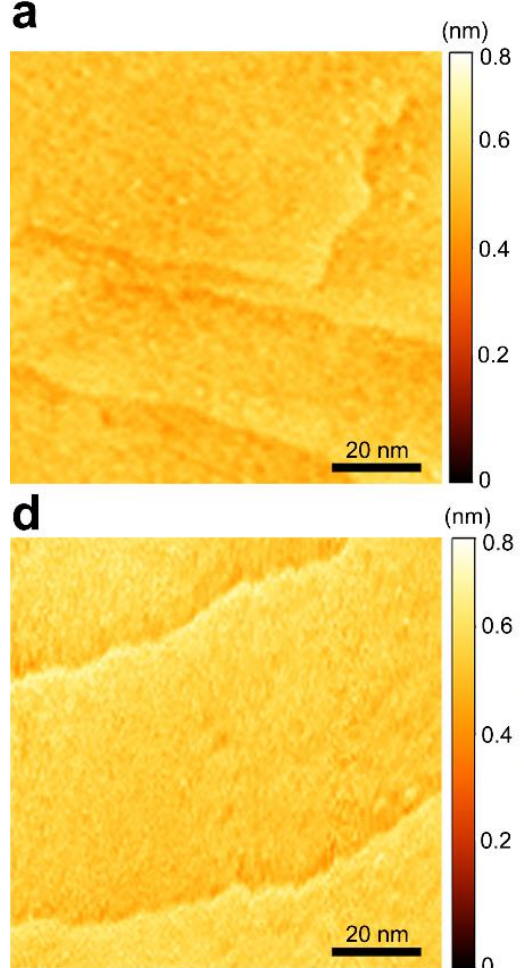

b
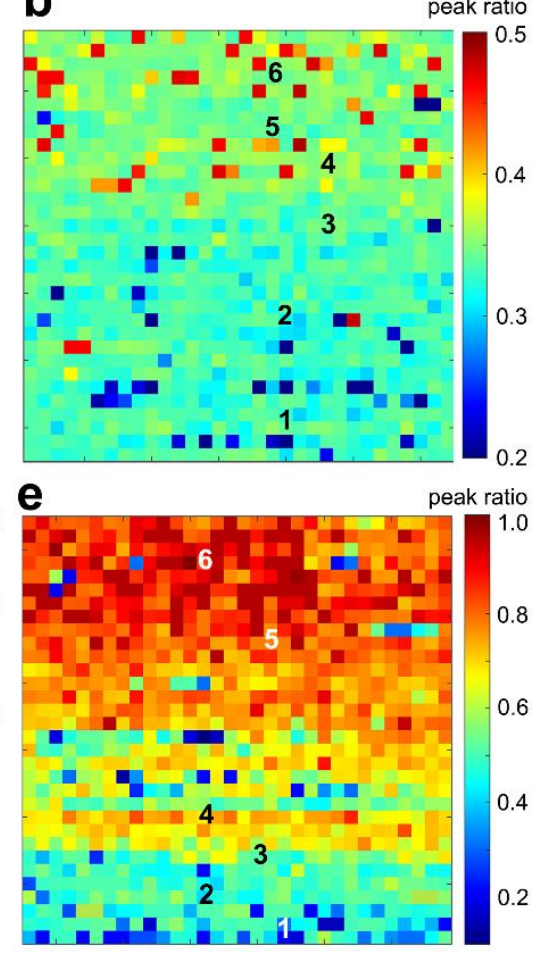

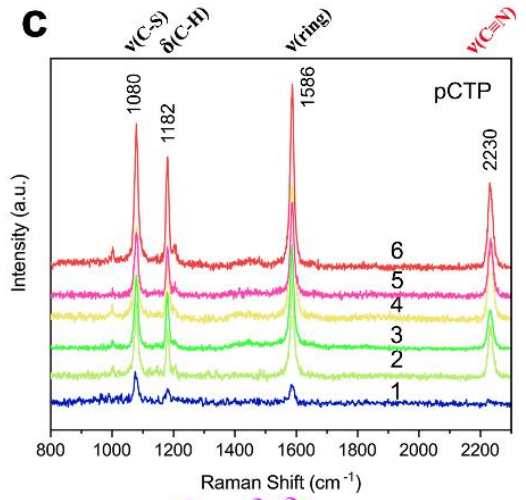

f

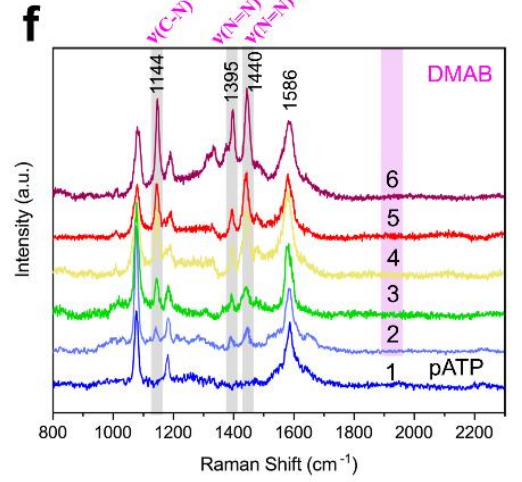

Figure 2. (a, d) Ex-situ STM images of (a) pCTP and (d) pATP pure SAMs on Au(111). (b, e) TERS intensity ratio images of (b) pCTP ( $I_{2230}$ to $\left.I_{1586}\right)$ and (e) DMAB ( $I_{1440}$ to $\left.I_{1586}\right)$ with different molecular components and orientation distributions. The dark blue pixels in $(b, e)$ represent unoccupied Au sites and/or unreacted pATP sites. All the maps were recorded over a $100 \times 100 \mathrm{~nm}^{2}$ area with $32 \times 32$ pixels on $\mathrm{Au}(111)$ from bottom to top. (c, f) Typical TERS spectra of (c) pCTP and (f) pATP/DMAB adsorbed in the locations marked with numbers in (b) and (e), respectively. The color coding in (c) and (f) represents how frequently each type of spectrum appears in the maps (b) and (e), respectively. 


\section{STM and TERS imaging of the binary SAMs}

The binary SAMs on Au(111) were prepared by coadsorption of pCPT and pATP (Figures 2a, d). Individual, fresh $\mathrm{Au}(111)$ substrates were immersed into an equimolar (1:1) mixed solution of these two thiolates at room temperature for different times: $10 \mathrm{~min}, 1 \mathrm{~h}, 2 \mathrm{~h}$, and $6 \mathrm{~h}$ (Figure 3). The morphologies of the binary SAMs on Au(111) were determined by STM under ambient conditions before TERS imaging (Figures 3a, c, e, g). Formation of a rougher surface and of vacancy islands can be observed on the binary SAMs covered $\mathrm{Au}(111)$ as the coadsorption time progresses; this is due to the extraction and mobility of surface Au atoms when thiolates assemble chemically into saturated SAMs towards the thermodynamically stable states. ${ }^{30,53}$ Compared to our previous work for benzenethiol/oligomeric phenylene-ethynylene thiol binary SAMs,${ }^{28}$ the upgraded TERS system with the acoustic isolation box that was used here clearly contributed to better STM/TERS imaging under ambient conditions (Figure S8). In addition, higher resolution STM imaging with mechanically cut Pt-Ir tips was capable of confirming the gradual changes of the surface morphology on $\mathrm{Au}(111)$ over time (Figure S9). Nevertheless, such STM imaging relying only on the height images cannot distinguish between pATP and pCTP on $\mathrm{Au}(111)$. 

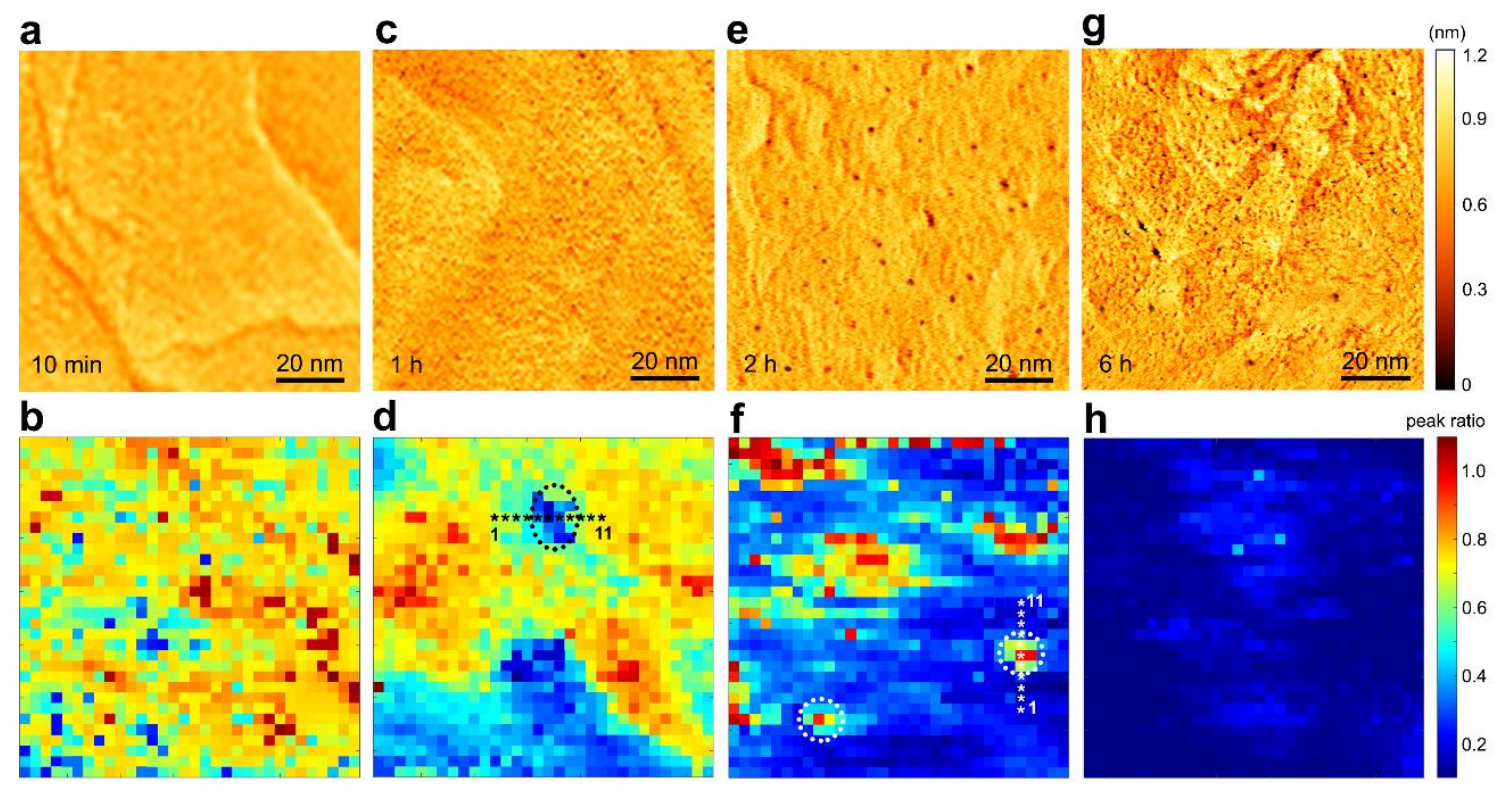

Figure 3. (a, c, e, g) Ex-situ STM images of pCTP/pATP binary SAMs on Au(111) over different coadsorption time. (a) $10 \mathrm{~min}$; (b) $1 \mathrm{~h}$; (c) $2 \mathrm{~h}$; (d) $6 \mathrm{~h}$. (b, d, f, h) Corresponding exsitu TERS intensity ratio images of DMAB/pCTP $\left(I_{1440}\right.$ to $\left.I_{2230}\right)$ with different molecular components and ratio distributions. The dark blue pixels represent the only pCTP and the hot pixels suggest both thiolates on $\mathrm{Au}(111)$. All the maps were recorded over a $100 \times 100 \mathrm{~nm}^{2}$ area with $32 \times 32$ pixels. The black circle in $(\mathrm{d})$ indicates a pure pCTP nanodomain $(\sim 10 \mathrm{~nm})$. The white circles in (f) indicate the binary nanodomains $(\sim 10 \mathrm{~nm})$. The stars marked with numbers in $(\mathrm{d}, \mathrm{f})$ are used for the spatial resolution analysis in Figure 4.

TERS intensity ratio ( $I_{1440}$ to $I_{2230}$ ) maps were also evaluated to assess the molecular component and spatial distribution of the binary SAMs ${ }^{34}$ as depicted in Figures $3 \mathrm{~b}, \mathrm{~d}, \mathrm{f}$, and $\mathrm{h}$. Since the $\mathrm{C} \equiv \mathrm{N}$ stretching mode at $2230 \mathrm{~cm}^{-1}$ for pCTP was visible in all the binary SAMs at all times, it was used as a standard peak to produce the intensity ratio maps. When the intensity ratio $\left(I_{1440}\right.$ to $\left.I_{2230}\right)$ dropped to less than ca. 0.2 (for more details, see Figure S10), these locations in the maps were assumed to be fully occupied by pCTP (Figures 3h). Otherwise, a mixed SAMs on $\mathrm{Au}(111)$ is present (Figure S10). Initially, after the first 10 min of coadsorption, both pCTP and pATP/DMAB can be found in the binary SAMs on Au(111) (Figure 3b). These binary thiolates are homogeneously mixed and randomly distributed on the surface via S-Au bonds, since the intensity ratios $\left(I_{1440}\right.$ to $\left.I_{2230}\right)$ all lie in the $0.6-0.8$ range. Fluctuations can be attributed to the variance of molecular orientation at room temperature. ${ }^{34}$ After $1 \mathrm{~h}$ 
coadsorption, a pure pCTP nanodomain (e.g., $<10 \mathrm{~nm}$, indicated by the dark blue pixels) was identified in Figure 3d, marked with a black circle, revealing that the binary SAM becomes segregated rather than intermixed at this stage. That is probably due to the different adsorption energy and subtle replacement competition between pCTP and pATP on Au(111) in methanol. Smaller and smaller binary nanodomains (e.g., $<10 \mathrm{~nm}$, indicated by the "hot" pixels) appeared in the TERS image after $2 \mathrm{~h}$ coadsorption (Figure $3 \mathrm{f}$ ). The increase of blue pixels in the map suggests the decrease of the surface coverage of pATP on Au(111) over time, i.e., pCTP gradually becomes the dominant adsorption species in the SAM during a longer coadsorption. Consequently, the replacement of pATP by pCTP on Au(111) occurs through the nucleation and extension of pCTP nanodomains over time (Figures 3b, d, f, h). Finally, as expected, no more pATP/DMAB (indicated by the hot pixels) was visible in the TERS map since adsorbed pATP has been fully replaced by pCTP after $6 \mathrm{~h}$ coadsorption (Figure $3 \mathrm{~h}$ ). 

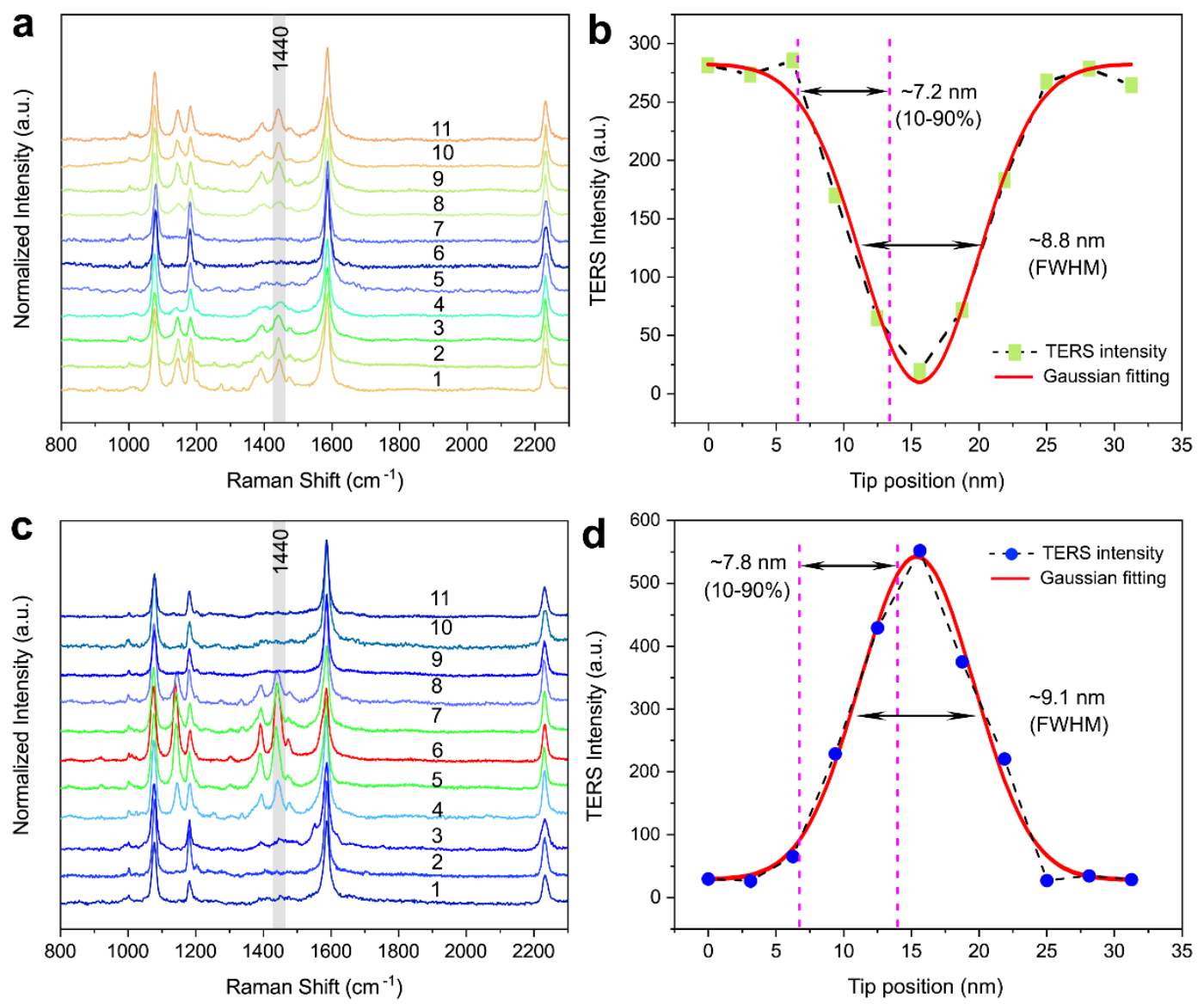

Figure 4. (a, c) Line-trace TERS spectra acquired from the (a) pure pCTP and (c) binary nanodomains along the black (Figure 3d) and white (Figure 3f) star lines, respectively. The color coding of the spectra in (a) and (c) is in line with their pixel colors in Figures $3 \mathrm{~d}$, f, respectively. (b, d) Corresponding TERS intensity and Gaussian fitting of the band at 1440 $\mathrm{cm}^{-1}$ in Figures 3d, f, respectively. The spatial resolution is estimated using a 10-90\% contrast criterion or using a full-width at half maximum (FWHM) analysis.

In order to estimate the spatial resolution under ambient conditions, visible nanodomains were chosen and extracted from the TERS maps of the binary SAM, as shown in Figures $3 \mathrm{~d}$, $\mathrm{f}$, indicated by black and white circles. The corresponding line-trace TERS spectra (black and white asterisks marked with numbers) acquired from these nanodomains indicate the TERS intensity variation of the $1440 \mathrm{~cm}^{-1}$ peak, offering an apparent contrast between the full pCTP areas and mixed pCTP/DMAB areas (Figures $4 \mathrm{a}, \mathrm{c})$. For the pure pCTP nanodomain in Figure $3 \mathrm{~d}$, the line profile analysis suggests a spatial resolution of $\sim 7.2 \mathrm{~nm}$ using the $10-90 \%$ criterion or $\sim 8.8 \mathrm{~nm}$ using the full-width at half maximum (FWHM) analysis (Figure $4 \mathrm{~b}$ ), respectively. 
For the binary nanodomains in Figure $3 \mathrm{f}$, the spatial resolutions are estimated to be $\sim 7.8 \mathrm{~nm}$ and $\sim 9.1 \mathrm{~nm}$ using the same methods, respectively. However, the real spatial resolution of our TERS imaging may in fact be underestimated because there are even smaller binary nanodomains that become discernible in the TERS map (Figure 3f).

\section{Adsorption energy in vacuum and solvent}

To further explore the coadsorption competition within the binary SAMs, we calculated the adsorption energy of pCTP and pATP on $\mathrm{Au}(111)$ using the DFT method, as depicted in Figure 5. Unlike a saturated surface coverage of benzenthiol (benzenthiol molecules : Au atoms $=$ 0.31) on $\mathrm{Au}(111)$ estimated by STM imaging, ${ }^{59}$ a lower surface coverage (1/4) of pCTP and pATP on $\mathrm{Au}(111)$ was applied in the current calculations due to a shorter coadsorption time. Namely, uniformly distributed pCTP or pATP molecules $(3 \times 3)$ were placed on the $\mathrm{Au}(111)$ surface $(6 \times 6$, Figures $S 11,12)$. When pCTP and pATP adsorb on Au(111) in vacuum, they energetically prefer the bridge-site binding with a S-Au bond length of $2.476 \AA$ and $2.493 \AA$, respectively (Figures 5c, d), similarly to previous reported DFT calculations. ${ }^{60,61}$ Moreover, due to the intermolecular and thiol/substrate interactions, ${ }^{62}$ the calculated lowest-energy structures suggest that their molecular planes tilted away by around $58^{\circ}(\mathrm{pCTP})$ and $55^{\circ}(\mathrm{pATP})$ from the surface normal (Figures 5c, d), respectively. Importantly, these energy calculations also reveal that pATP $(-0.82 \mathrm{eV}$, Table S2) anchors on Au(111) with lower adsorption energy than that of pCTP $(-0.62 \mathrm{eV}$, Tables $\mathrm{S} 2,3)$ in vacuum. Such calculated energies are also close to the reported $\mathrm{S}-\mathrm{Au}$ binding energies for the alkanethiol $(74 \mathrm{~kJ} / \mathrm{mol}, 0.77 \mathrm{eV})$ and arenethiol $(46 \mathrm{~kJ} / \mathrm{mol}, 0.48 \mathrm{eV}) .{ }^{63}$ The more negative adsorption energy of pATP implies that it is supposed to have stronger binding than pCTP on Au(111) in vacuum, and thus pATP would 
prevail in the coadsorption competition. ${ }^{64}$ However, this assumption is in contrast to our experimental observations (Figure 3).

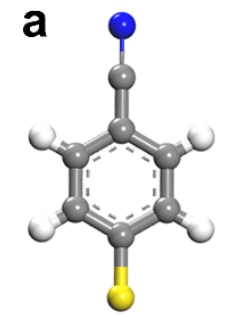

pCTP

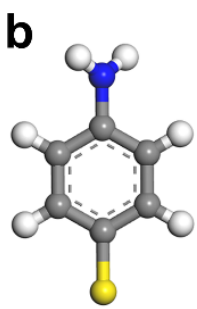

pATP

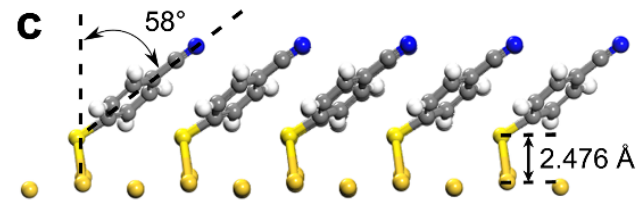

- 8 0 0 80808 0 Au
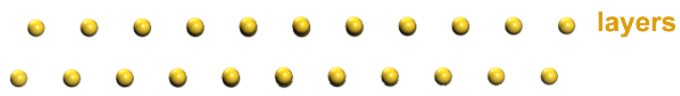

$$
\left(\Delta E_{\mathrm{ads}}=-0.62 \mathrm{eV}\right)
$$

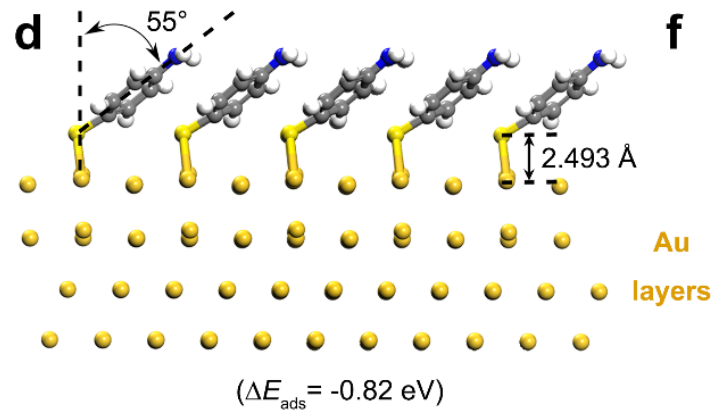

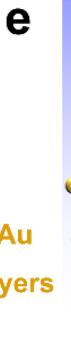
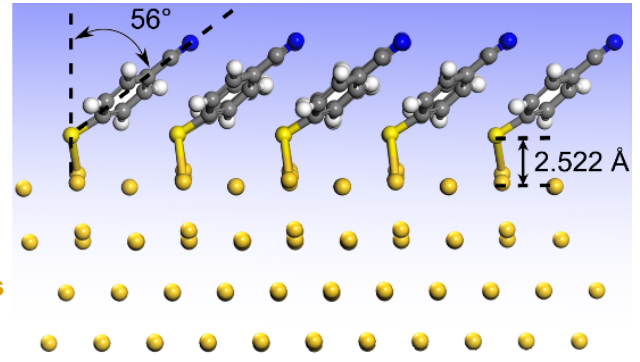

$\left(\Delta E_{\mathrm{ads}}=-0.92 \mathrm{eV}\right)$

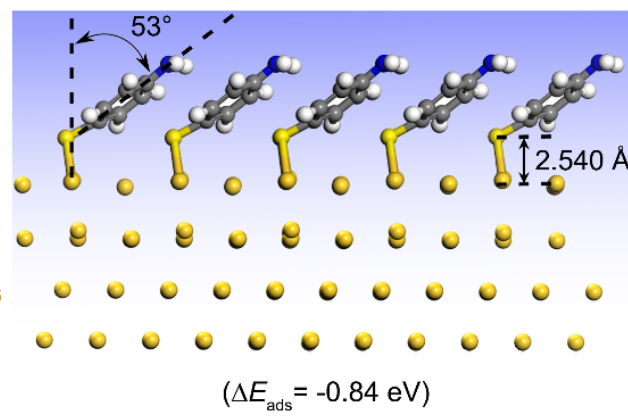

Figure 5. (a, b) Chemical structures of (a) pCTP and (b) pATP adsorbed on Au(111). (c, d) Optimized configurations of (c) pCTP and (d) pATP adsorbed on Au(111) in vacuum. (e, f) Optimized configurations of (e) pCTP and (f) pATP adsorbed on Au(111) in methanol. The corresponding adsorption energies for individual thiolates, the S-Au bond distances, and the relative angles between the molecular plane and the surface normal are marked in the respective systems.

To understand this discrepancy between the experimental and calculated results, implicit solvation models have been taken into account in further calculations. In the continuum SCCS models, the solvent effects are included implicitly as a dielectric medium. ${ }^{41}$ Compared to the adsorption in vacuum (Figures 5c, d), pCTP and pATP share similar S-Au bond lengths and molecule-surface tilted angles on $\mathrm{Au}(111)$ in methanol (Figures 5e, f). On the contrary, they tend to adsorb on $\mathrm{Au}(111)$ with different adsorption energies of $-0.92 \mathrm{eV}(\mathrm{pCTP})$ and -0.84 eV (pATP) in methanol (Tables S2, S3), respectively. These results suggest that pCTP has stronger binding than pATP on $\mathrm{Au}(111)$ in methanol, thereby gradually replacing pATP SAMs 
during their coadsorption under the same conditions, which is in good agreement with the TERS observations (Figure 3). One straightforward explanation is that the solvation effect plays a crucial role in the coadsorption competition of the thiolate SAMs. Moreover, owing to the different polarity and dipole moment between benzonitrile $(\mathrm{PhCN})$ and aniline $\left(\mathrm{PhNH}_{2}\right),{ }^{65}$, ${ }^{66}$ the polar solvent (e.g., methanol) shows more significant solvation effects on pATP with higher polarity and thus better ability to dissolve pATP to the bulk solvent. Alternatively, dissolved pCTP with lower polarity is likely to adsorb and form nucleations on $\mathrm{Au}(111)$ and then extend these nanodomains over time. Consequently, pCTP can gradually replace pATP on $\mathrm{Au}(111)$ during the coadsorption.

\section{CONCLUSIONS}

In summary, we utilized TERS to provide spectroscopic imaging of binary thiolate SAMs on $\mathrm{Au}(111)$ at the nanometer scale and molecular level under ambient conditions, and the experimental results were further supported by DFT calculations. Two structurally similar aromatic thiolates (pCTP and pATP) with different tail groups (-CN versus $-\mathrm{NH}_{2}$ ) were selected as coadsorption competitors on $\mathrm{Au}(111)$ in an equimolar mixed methanol solution. TERS mapping revealed that pCTP and pATP can initially coadsorb on Au(111) randomly and homogeneously; then pCTP will gradually replace pATP through the extension of pCTP nanodomains as the immersion time progresses, in the case where STM images were only visible for the change in surface topography but "blind" for the change in chemical information. Additionally, under the STM-TERS with the Ag tip/Au substrate configuration, TERS imaging also allowed for visualization of the plasmon-induced photocatalysis of pATP to DMAB and the phase segregation within the mixed SAMs with a spatial resolution of less than $9 \mathrm{~nm}$ under ambient conditions. Combined with DFT calculations that take implicit solvation into account, 
we found out that the adsorption energy of individual pCTP and pATP on Au(111) in vacuum was estimated to be ca. $-0.62 \mathrm{eV}$ and $-0.82 \mathrm{eV}$, respectively. However, this energy contrast was reversed for pCTP $(-0.92 \mathrm{eV})$ and pATP $(-0.84 \mathrm{eV})$ in methanol, suggesting that pCTP exhibits stronger adsorption than pATP at the $\mathrm{Au}(111) /$ methanol interface, and the solvation effect plays a vital role in coadsorption behavior of the binary SAMs. This work offers a viable way to chemically distinguish mixed SAMs at the nanoscale and opens prospects for a better understanding of the roles, effects, and importance of molecular level mixing in components for molecular electronics and heterogeneous catalysis.

\section{ASSOCIATED CONTENT}

*Supporting Information

The Supporting Information is available free of charge at $\mathrm{xxxxx}$

- Schematic illustration of the TERS setup; photo of the acoustic isolation box; highresolution STM images of the bare $\mathrm{Au}(111)$ and binary SAMs; time-dependent TER spectral evolution of pATP and DMAB; SEM image of the Ag tip; assignments of the selected Raman peaks; top view of the thiolate optimized configurations; tables of the adsorption energy calculations

\section{AUTHOR INFORMATION}

\section{Corresponding Authors}

Feng Shao - Department of Physics and Astronomy, National Graphene Institute, University of Manchester, Manchester, M13 9PL, UK; orcid.org/0000-0003-3879-5884; Email: feng.shao@manchester.ac.uk 
Renato Zenobi - Department of Chemistry and Applied Biosciences, ETH Zurich, 8093, Zurich, Switzerland; orcid.org/0000-0001-5211-4358. Email: zenobi@org.chem.ethz.ch

\section{Authors}

Liqing Zheng - Department of Chemistry and Applied Biosciences, ETH Zurich, 8093 Zurich, Switzerland; orcid.org/0000-0001-7848-6985.

Jinggang Lan - Department of Chemistry, University of Zurich, 8057 Zurich, Switzerland; orcid.org/0000-0001-6353-2539.

\section{ACKNOWLEDGMENTS}

This research was supported by the Marie-Skłodowska-Curie Individual Fellowship (8416532DvdWHs, to F.S.) and the European Research Council grant (741431-2DNanoSpec, to R.Z.). We thank the High Performance Computing Team at ETH Zurich for help with DFT calculations.

\section{Notes}

The authors declare no competing financial interest. 


\section{REFERENCES}

(1) Love, J. C.; Estroff, L. A.; Kriebel, J. K.; Nuzzo, R. G.; Whitesides, G. M., SelfAssembled Monolayers of Thiolates on Metals as a Form of Nanotechnology. Chem. Rev. 2005, 105 (4), 1103-1170.

(2) Häkkinen, H., The gold-sulfur interface at the nanoscale. Nat. Chem. 2012, 4 (6), 443-455.

(3) Reimers, J. R.; Ford, M. J.; Halder, A.; Ulstrup, J.; Hush, N. S., Gold surfaces and nanoparticles are protected by $\mathrm{Au}(0)$-thiyl species and are destroyed when $\mathrm{Au}(\mathrm{I})$-thiolates form. Proc. Natl. Acad. Sci. U.S.A. 2016, 113 (11), E1424.

(4) Kim, J.; Rim, Y. S.; Liu, Y.; Serino, A. C.; Thomas, J. C.; Chen, H.; Yang, Y.; Weiss, P. S., Interface Control in Org. Electron. Using Mixed Monolayers of Carboranethiol Isomers. Nano Lett. 2014, 14 (5), 2946-2951.

(5) Asyuda, A.; Wan, X.; Zharnikov, M., Binary aromatic self-assembled monolayers: electrostatic properties and charge tunneling rates across the molecular framework. Phys. Chem. Chem. Phys. 2020, 22 (19), 10957-10967.

(6) Katsouras, I.; Geskin, V.; Kronemeijer, A. J.; Blom, P. W. M.; de Leeuw, D. M., Binary self-assembled monolayers: Apparent exponential dependence of resistance on average molecular length. Org. Electron. 2011, 12 (5), 857-864.

(7) Majumdar, S.; Malen, J. A.; McGaughey, A. J. H., Cooperative Molecular Behavior Enhances the Thermal Conductance of Binary Self-Assembled Monolayer Junctions. Nano Lett. 2017, 17 (1), 220-227.

(8) Pieters, G.; Prins, L. J., Catalytic self-assembled monolayers on gold nanoparticles. New J. Chem. 2012, 36 (10), 1931-1939.

(9) Kuna, J. J.; Voïtchovsky, K.; Singh, C.; Jiang, H.; Mwenifumbo, S.; Ghorai, P. K.; Stevens, M. M.; Glotzer, S. C.; Stellacci, F., The effect of nanometre-scale structure on interfacial energy. Nat. Mater. 2009, 8 (10), 837-842.

(10) Kong, G. D.; Byeon, S. E.; Park, S.; Song, H.; Kim, S.-Y.; Yoon, H. J., Mixed Molecular Electronics: Tunneling Behaviors and Applications of Mixed Self-Assembled Monolayers. $A d v$. Electron. Mater. 2020, 6 (2), 1901157.

(11) Ooi, Y.; Hobara, D.; Yamamoto, M.; Kakiuchi, T., Ideal Nonideality in Adsorption of 2-Aminoethanethiol and 2-Mercaptoethane Sulfonic Acid To Form Electrostatically Stabilized Binary Self-Assembled Monolayers on Au(111). Langmuir 2005, 21 (24), 11185-11189.

(12) Heister, K.; Zharnikov, M.; Grunze, M.; Johansson, L. S. O., Adsorption of Alkanethiols and Biphenylthiols on $\mathrm{Au}$ and Ag Substrates: A High-Resolution X-ray Photoelectron Spectroscopy Study. J. Phys. Chem. B 2001, 105 (19), 4058-4061.

(13) Ortiz de la Morena, R.; Asyuda, A.; Lu, H.; Aitchison, H.; Turner, K.; Francis, S. M.; Zharnikov, M.; Buck, M., Shape controlled assembly of carboxylic acids: formation of a binary monolayer by intercalation into molecular nanotunnels. Phys. Chem. Chem. Phys. 2020, 22 (7), 4205-4215.

(14) Hobara, D.; Uno, Y.; Kakiuchi, T., Immobilization of horseradish peroxidase on nanometre-scale domains of binary self-assembled monolayers formed from dithiobis-Nsuccinimidyl propionate and 1-tetradecanethiol on Au(111). Phys. Chem. Chem. Phys. 2001, 3 (16), 3437-3441.

(15) Smith, R. K.; Reed, S. M.; Lewis, P. A.; Monnell, J. D.; Clegg, R. S.; Kelly, K. F.; Bumm, L. A.; Hutchison, J. E.; Weiss, P. S., Phase Separation within a Binary Self-Assembled Monolayer on $\mathrm{Au}\{111\}$ Driven by an Amide-Containing Alkanethiol. J. Phys. Chem. B 2001, 105 (6), 1119-1122.

(16) Hayes, W. A.; Kim, H.; Yue, X.; Perry, S. S.; Shannon, C., Nanometer-Scale Patterning of Surfaces Using Self-Assembly Chemistry. 2. Preparation, Characterization, and 
Electrochemical Behavior of Two-Component Organothiol Monolayers on Gold Surfaces. Langmuir 1997, 13 (9), 2511-2518.

(17) Guo, Q.; Li, F., Self-assembled alkanethiol monolayers on gold surfaces: resolving the complex structure at the interface by STM. Phys. Chem. Chem. Phys. 2014, 16 (36), 1907419090.

(18) Wang, Y.; Chi, Q.; Zhang, J.; Hush, N. S.; Reimers, J. R.; Ulstrup, J., Chain-Branching Control of the Atomic Structure of Alkanethiol-Based Gold-Sulfur Interfaces. J. Am. Chem. Soc. 2011, 133 (38), 14856-14859.

(19) Yan, J.; Ouyang, R.; Jensen, P. S.; Ascic, E.; Tanner, D.; Mao, B.; Zhang, J.; Tang, C.; Hush, N. S.; Ulstrup, J.; Reimers, J. R., Controlling the Stereochemistry and Regularity of Butanethiol Self-Assembled Monolayers on Au(111). J. Am. Chem. Soc. 2014, 136 (49), 17087-17094.

(20) Mannsfeld, S. C. B.; Canzler, T. W.; Fritz, T.; Proehl, H.; Leo, K.; Stumpf, S.; Goretzki, G.; Gloe, K., The Structure of [4-(Phenylazo)phenoxy]hexane-1-thiol Self-Assembled Monolayers on Au(111). J. Phys. Chem. B 2002, 106 (9), 2255-2260.

(21) Schmid, T.; Opilik, L.; Blum, C.; Zenobi, R., Nanoscale Chemical Imaging Using TipEnhanced Raman Spectroscopy: A Critical Review. Angew. Chem. Int. Ed. 2013, 52 (23), 5940-5954.

(22) Pozzi, E. A.; Goubert, G.; Chiang, N.; Jiang, N.; Chapman, C. T.; McAnally, M. O.; Henry, A.-I.; Seideman, T.; Schatz, G. C.; Hersam, M. C.; Duyne, R. P. V., UltrahighVacuum Tip-Enhanced Raman Spectroscopy. Chem. Rev. 2017, 117 (7), 4961-4982.

(23) Wang, X.; Huang, S.-C.; Huang, T.-X.; Su, H.-S.; Zhong, J.-H.; Zeng, Z.-C.; Li, M.H.; Ren, B., Tip-enhanced Raman spectroscopy for surfaces and interfaces. Chem. Soc. Rev. 2017, 46 (13), 4020-4041.

(24) Richard-Lacroix, M.; Zhang, Y.; Dong, Z.; Deckert, V., Mastering high resolution tipenhanced Raman spectroscopy: towards a shift of perception. Chem. Soc. Rev. 2017, 46 (13), 3922-3944.

(25) Picardi, G.; Chaigneau, M.; Ossikovski, R.; Licitra, C.; Delapierre, G., Tip enhanced Raman spectroscopy on azobenzene thiol self-assembled monolayers on Au(111). J. Raman Spectrosc. 2009, 40 (10), 1407-1412.

(26) Stadler, J.; Schmid, T.; Opilik, L.; Kuhn, P.; Dittrich, P. S.; Zenobi, R., Tip-enhanced Raman spectroscopic imaging of patterned thiol monolayers. Beilstein J. Nanotechnol. 2011, 2, 509-515.

(27) Picardi, G.; Królikowska, A.; Yasukuni, R.; Chaigneau, M.; Escude, M.; Mourier, V.; Licitra, C.; Ossikovski, R., Exchange of Methyl- and Azobenzene-Terminated Alkanethiols on Polycrystalline Gold Studied by Tip-Enhanced Raman Mapping. ChemPhysChem 2014, 15 (2), 276-282.

(28) Lin, W.-I.; Shao, F.; Stephanidis, B.; Zenobi, R., Tip-enhanced Raman spectroscopic imaging shows segregation within binary self-assembled thiol monolayers at ambient conditions. Anal. Bioanal.Chem. 2015, 407 (27), 8197-8204.

(29) Horimoto, N. N.; Tomizawa, S.; Fujita, Y.; Kajimoto, S.; Fukumura, H., Nano-scale characterization of binary self-assembled monolayers under an ambient condition with STM and TERS. Chem. Commun. 2014, 50 (69), 9862-9864.

(30) Hong, M.; Yokota, Y.; Hayazawa, N.; Kazuma, E.; Kim, Y., Homogeneous Dispersion of Aromatic Thiolates in the Binary Self-Assembled Monolayer on $\mathrm{Au}(111)$ via Displacement Revealed by Tip-Enhanced Raman Spectroscopy. J. Phys. Chem. C 2020, 124 (24), 1314113149.

(31) Nazmutdinov, R. R.; Zhang, J.; Zinkicheva, T. T.; Manyurov, I. R.; Ulstrup, J., Adsorption and in situ scanning tunneling microscopy of cysteine on Au (111): Structure, energy, and tunneling contrasts. Langmuir 2006, 22 (18), 7556-7567. 
(32) Granda-Marulanda, L. P.; Builes, S.; Koper, M. T. M.; Calle-Vallejo, F., Influence of Van der Waals Interactions on the Solvation Energies of Adsorbates at Pt-Based Electrocatalysts. ChemPhysChem 2019, 20 (22), 2968-2972.

(33) Rendón-Calle, A.; Builes, S.; Calle-Vallejo, F., Substantial improvement of electrocatalytic predictions by systematic assessment of solvent effects on adsorption energies. Appl. Catal., B 2020, 276, 119147.

(34) Shao, F.; Dai, W.; Zhang, Y.; Zhang, W.; Schlüter, A. D.; Zenobi, R., Chemical Mapping of Nanodefects within 2D Covalent Monolayers by Tip-Enhanced Raman Spectroscopy. ACS Nano 2018, 12 (5), 5021-5029.

(35) Shao, F.; Müller, V.; Zhang, Y.; Schlüter, A. D.; Zenobi, R., Nanoscale Chemical Imaging of Interfacial Monolayers by Tip-Enhanced Raman Spectroscopy. Angew. Chem. Int. Ed. 2017, 56 (32), 9361-9366.

(36) Shao, F.; Wang, W.; Yang, W.; Yang, Z.; Zhang, Y.; Lan, J.; Dieter Schlüter, A.; Zenobi, R., In-situ nanospectroscopic imaging of plasmon-induced two-dimensional [4+4]cycloaddition polymerization on $\mathrm{Au}(111)$. Nat. Commun. 2021, 12 (1), 4557.

(37) Shao, F.; Zenobi, R., Tip-enhanced Raman spectroscopy: principles, practice, and applications to nanospectroscopic imaging of 2D materials. Anal. Bioanal.Chem. 2019, 411 (1), 37-61.

(38) Wang, X.; Zhong, J.-H.; Zhang, M.; Liu, Z.; Wu, D.-Y.; Ren, B., Revealing Intermolecular Interaction and Surface Restructuring of an Aromatic Thiol Assembling on $\mathrm{Au}(111)$ by Tip-Enhanced Raman Spectroscopy. Anal. Chem. 2016, 88 (1), 915-921.

(39) Neese, F., Prediction of molecular properties and molecular spectroscopy with density functional theory: From fundamental theory to exchange-coupling. Coord. Chem. Rev. 2009, 253 (5), 526-563.

(40) Hutter, J.; Iannuzzi, M.; Schiffmann, F.; VandeVondele, J., cp2k: atomistic simulations of condensed matter systems. WIREs Comput. Mol. Sci. 2014, 4 (1), 15-25.

(41) Kühne, T. D.; Iannuzzi, M.; Del Ben, M.; Rybkin, V. V.; Seewald, P.; Stein, F.; Laino, T.; Khaliullin, R. Z.; Schütt, O.; Schiffmann, F.; Golze, D.; Wilhelm, J.; Chulkov, S.; BaniHashemian, M. H.; Weber, V.; Borštnik, U.; Taillefumier, M.; Jakobovits, A. S.; Lazzaro, A.; Pabst, H.; Müller, T.; Schade, R.; Guidon, M.; Andermatt, S.; Holmberg, N.; Schenter, G. K.; Hehn, A.; Bussy, A.; Belleflamme, F.; Tabacchi, G.; Glöß, A.; Lass, M.; Bethune, I.; Mundy, C. J.; Plessl, C.; Watkins, M.; VandeVondele, J.; Krack, M.; Hutter, J., CP2K: An electronic structure and molecular dynamics software package - Quickstep: Efficient and accurate electronic structure calculations. J. Chem. Phys. 2020, 152 (19), 194103.

(42) Perdew, J. P.; Burke, K.; Ernzerhof, M., Generalized Gradient Approximation Made Simple. Phys. Rev. Lett. 1996, 77 (18), 3865-3868.

(43) Grimme, S., Semiempirical GGA-type density functional constructed with a long-range dispersion correction. J. Comput. Chem. 2006, 27 (15), 1787-1799.

(44) Zheng, L.-Q.; Yang, S.; Lan, J.; Gyr, L.; Goubert, G.; Qian, H.; Aprahamian, I.; Zenobi, R., Solution Phase and Surface Photoisomerization of a Hydrazone Switch with a Long Thermal Half-Life. J. Am. Chem. Soc. 2019, 141 (44), 17637-17645.

(45) VandeVondele, J.; Hutter, J., Gaussian basis sets for accurate calculations on molecular systems in gas and condensed phases. J. Chem. Phys. 2007, 127 (11), 114105.

(46) Hartwigsen, C.; Goedecker, S.; Hutter, J., Relativistic separable dual-space Gaussian pseudopotentials from H to Rn. Phys. Rev. B 1998, 58 (7), 3641-3662.

(47) Rutter, M. J., Charged surfaces and slabs in periodic boundary conditions. Electron. Struct. 2021, 3 (1), 015002.

(48) Andreussi, O.; Dabo, I.; Marzari, N., Revised self-consistent continuum solvation in electronic-structure calculations. J. Chem. Phys. 2012, 136 (6), 064102. 
(49) Sementa, L.; Andreussi, O.; Goddard Iii, W. A.; Fortunelli, A., Catalytic activity of Pt38 in the oxygen reduction reaction from first-principles simulations. Catal. Sci. Technol. 2016, 6 (18), 6901-6909.

(50) Fattebert, J.-L.; Gygi, F., Density functional theory for efficient ab initio molecular dynamics simulations in solution. J. Comput. Chem. 2002, 23 (6), 662-666.

(51) Sachdeva, S.; Venkatesh, M. R.; Mansouri, B. E.; Wei, J.; Bossche, A.; Kapteijn, F.; Zhang, G. Q.; Gascon, J.; de Smet, L. C. P. M.; Sudhölter, E. J. R., Sensitive and Reversible Detection of Methanol and Water Vapor by In Situ Electrochemically Grown CuBTC MOFs on Interdigitated Electrodes. Small 2017, 13 (29), 1604150.

(52) Gurdal, Y., Aromatic versus aliphatic thiols on Au(111) surface: a DFT exploration of adsorption registry and electronic structure. Mol. Simul. 2020, 46 (1), 22-32.

(53) Sun, J.-J.; Su, H.-S.; Yue, H.-L.; Huang, S.-C.; Huang, T.-X.; Hu, S.; Sartin, M. M.; Cheng, J.; Ren, B., Role of Adsorption Orientation in Surface Plasmon-Driven Coupling Reactions Studied by Tip-Enhanced Raman Spectroscopy. J. Phys. Chem. Lett. 2019, 10 (10), 2306-2312.

(54) Sun, M.; Xu, H., A Novel Application of Plasmonics: Plasmon-Driven Surface-Catalyzed Reactions. Small 2012, 8 (18), 2777-2786.

(55) Wu, D.-Y.; Liu, X.-M.; Huang, Y.-F.; Ren, B.; Xu, X.; Tian, Z.-Q., Surface Catalytic Coupling Reaction of p-Mercaptoaniline Linking to Silver Nanostructures Responsible for Abnormal SERS Enhancement: A DFT Study. J. Phys. Chem. C 2009, 113 (42), 18212-18222. (56) Wu, D.-Y.; Zhao, L.-B.; Liu, X.-M.; Huang, R.; Huang, Y.-F.; Ren, B.; Tian, Z.-Q., Photon-driven charge transfer and photocatalysis of p-aminothiophenol in metal nanogaps: a DFT study of SERS. Chem. Commun. 2011, 47 (9), 2520-2522.

(57) Huang, Y.-F.; Wu, D.-Y.; Zhu, H.-P.; Zhao, L.-B.; Liu, G.-K.; Ren, B.; Tian, Z.-Q., Surface-enhanced Raman spectroscopic study of p-aminothiophenol. Phys. Chem. Chem. Phys. 2012, 14 (24), 8485-8497.

(58) Kutsenko, V. Y.; Lopatina, Y. Y.; Bossard-Giannesini, L.; Marchenko, O. A.; Pluchery, O.; Snegir, S. V., Alkylthiol self-assembled monolayers on $\mathrm{Au}(111)$ with tailored tail groups for attaching gold nanoparticles. Nanotechnology 2017, 28 (23), 235603.

(59) Wan, L.-J.; Terashima, M.; Noda, H.; Osawa, M., Molecular Orientation and Ordered Structure of Benzenethiol Adsorbed on Gold(111). J. Phys. Chem. B 2000, 104 (15), 35633569.

(60) Ting, E. C.; Popa, T.; Paci, I., Surface-site reactivity in small-molecule adsorption: A theoretical study of thiol binding on multi-coordinated gold clusters. Beilstein J. Nanotechnol. 2016, 7 (1), 53-61.

(61) Pakiari, A. H.; Jamshidi, Z., Nature and Strength of $\mathrm{M}-\mathrm{S}$ Bonds $(\mathrm{M}=\mathrm{Au}, \mathrm{Ag}$, and $\mathrm{Cu})$ in Binary Alloy Gold Clusters. J. Phys. Chem. A 2010, 114 (34), 9212-9221.

(62) Frey, S.; Stadler, V.; Heister, K.; Eck, W.; Zharnikov, M.; Grunze, M.; Zeysing, B.; Terfort, A., Structure of Thioaromatic Self-Assembled Monolayers on Gold and Silver. Langmuir 2001, 17 (8), 2408-2415.

(63) Yang, G.; Liu, G.-y., New Insights for Self-Assembled Monolayers of Organothiols on $\mathrm{Au}(111)$ Revealed by Scanning Tunneling Microscopy. J. Phys. Chem. B 2003, 107 (34), 87468759.

(64) Xu, L.; Lin, J.; Bai, Y.; Mavrikakis, M., Atomic and Molecular Adsorption on Cu(111). Top. Catal. 2018, 61 (9), 736-750.

(65) Reichardt, C.; Welton, T., Solvents and Solvent Effects in Organic Chemistry, 4th ed.; Wiley-VCH: Weinheim, 2011.

(66) Iyemperumal, S. K.; Deskins, N. A., Evaluating Solvent Effects at the Aqueous/Pt(111) Interface. ChemPhysChem 2017, 18 (16), 2171-2190. 


\section{TOC}

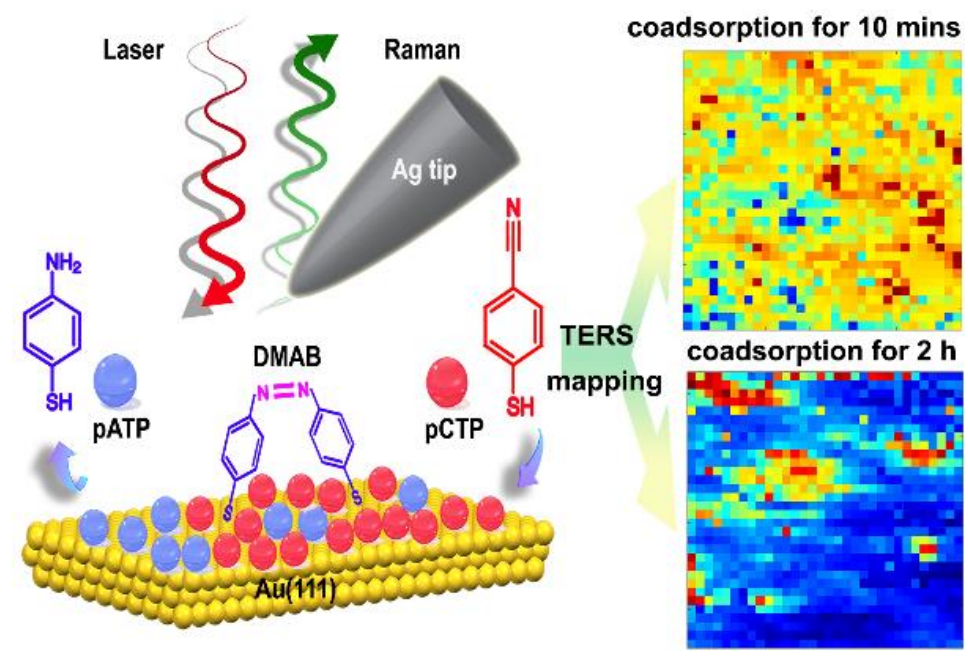

